\title{
PDE BASED HISTOGRAM MODIFICATION WITH EMBEDDED MORPHOLOGICAL PROCESSING OF THE LEVEL-SETS
}

\author{
GY. CSEREY, CS. REKECZKY, AND P. FÖLDESY \\ Analogical and Neural Computing Systems Laboratory, \\ Computer and Automation Research Institute, \\ Hungarian Academy of Sciences, Budapest, Hungary
}

\begin{abstract}
This paper describes parallel histogram modification techniques with embedded morphological preprocessing methods within the CNN-UM framework. The procedure is formulated in terms of nonlinear partial differential equations (PDE) and approximated through finite differences in space, resulting in coupled nonlinear ordinary differential equations (ODE). The I/O mapping of the system (containing both local and global couplings) can be calculated by a complex analogic (analog and logic) algorithm executed on a stored program nonlinear array processor, called the cellular nonlinear network universal machine (CNN-UM, [3]). We describe and illustrate how the implementation of the algorithm results in an adaptive multi-thresholding scheme when histogram modification is combined with embedded morphological processing at a finite (low) number of grayscale levels. This has obvious advantages if the further processing steps are segmentation and/or recognition. Experimental results processing real-life and echocardiography images are measured on different hardware/software platforms, including a 64x64 CNN-UM chip (ACE4k, [6]).
\end{abstract}

\section{Introduction}

In this paper we analyze a set of PDEs designed for simultaneous contrast enhancement, noise suppression and shape enhancement. Based on spatial approximations and using a discrete set of gray-values these PDEs will be decomposed to spatially interacting ODEs (discrete in space and continuous in time). The resulting analogic (analog and logic) CNN ([1]-[6]) algorithms are implemented on different hardware-software platforms. Measurement results prove that various process real-time image processing based solutions could use this technique as an efficient front-end scheme. Furthermore, it is demonstrated that the implementations accelerated by a CNN-UM chip [6] are superior to the optimized solutions on a high-end DSP (Texas 6x).

\section{Contrast Enhancement through Histogram Modification}

Due to poor or changing lighting conditions image snapshots (or video-flows) are often captured at low contrast in different scenarios. On the other hand a number of algorithms taking these images as inputs are in general fairly sensitive to large (or rapid) contrast changes that could significantly degrade the processing performance. Recent advancements of different visual microprocessors resulted in new hardware platforms for parallel algorithms and these are also the basis for developing new parallel nonlinear techniques for contrast improvement.

The most common way to improve the contrast of an image is to modify its grayvalue distribution, the image histogram. For example, histogram equalization is a basic method that drives the image pixel values into different gray-scale levels in order to achieve a uniform distribution [10],[11]. This global technique improves the contrast and does not modify the level-sets (the family of binary shadows of the image). Histogram modifications can also be formulated in terms of PDEs [12]-[14] that gives hope to a fully parallel implementation. In this paper we explore and modify some of these recent

HistModif\&MorphProc_CNNA2002 submitted to World Scientific : 4/26/2002 : 2:11 PM 
formulations, we derive and implement efficient new algorithms for cellular nonlinear array processor architectures.

\section{PDE formulations}

Let us consider the following PDE designed for histogram equalization ([12]-[14]):

$$
\frac{\partial \Phi(x, y, t)}{\partial t}=\left(N^{2}-\frac{N^{2}}{M} \Phi(x, y, t)\right)-A\left[L(v, w, t)_{\Phi(x, y, t)}\right]
$$

where $\Phi(x, y, t):[0, N]^{2} \mathrm{x}[0, T] \rightarrow[0, M]$ is the image intensity, $N$ and $M$ are constants, and $A[]:. \mathrm{R}^{2} \rightarrow \mathrm{R}$ stands for the area measure of a level-set.

The level-sets $L(v, w, t)_{\phi(x, y, t)}:[0, N]^{2} \times[0, T] \rightarrow\{0, M\}$ are the "binary shadows" of the image and can be described as follows:

$$
L(v, w, t)_{\Phi(x, y, t)}=L_{\Phi}= \begin{cases}1, & \text { if } \Phi(v, w, t) \geq \Phi(x, y, t) \\ 0 & \text { otherwise }\end{cases}
$$

Then the calculation of the area measure is defined as follows:

$$
A\left[L_{\Phi}\right]=A[(v, w): \Phi(v, w, t) \geq \Phi(x, y, t)]=\int_{v=0}^{N} \int_{w=0}^{N} L_{\Phi} d v d w
$$

A modified and extended version of (3) that allows a simultaneous contrast enhancement with noise suppression ([12]-[14]) is as follows:

$$
\frac{\partial \Phi(x, y, t)}{\partial t}=\alpha \kappa+\left(N^{2}-H(\Phi(x, y, t))\right)-A\left[L_{\Phi}\right]
$$

where $\alpha$ is a constant, $\kappa$ is a regularizing term, and $H():. \mathrm{R} \rightarrow \mathrm{R}$ represents a prescribed monotone increasing function.

A further novel generalization that adds shape enhancement by morphological processing of the level-sets is as follows:

$$
\frac{\partial \Phi(x, y, t)}{\partial t}=\alpha \kappa+\left(N^{2}-H(\Phi(x, y, t))\right)-A\left[L_{g(\Phi)}\right]
$$

where $L_{\mathrm{g}(\Phi)}$ is a "threshold transformed" level set and $g():. \mathrm{R} \rightarrow \mathrm{R}$ is a general nonlinear function.

We make the function $g($.$) to depend implicitly (explained later) on \Gamma[U, B]$ that stands for a morphological processing of $U$ with a structuring element $B$ (a disk).

Two forms of morphological processing will be investigated, the n-step "erodedilate" and "dilate-erode" operations $(\oplus$ and $\otimes$ stand for dilation and erosion, respectively) described by the following set-theoretic formulations:

$$
\Gamma[U, B]=\left((U \oplus B)^{(n)} \otimes\right)^{(n)} \vee \quad \Gamma[U, B]=\left((U \otimes B)^{(n)} \oplus\right)^{(n)}
$$

The input set $U$ of these operations is either a level-set $L_{\Phi}$ or a level-set section: $L_{\Phi 1, \Phi 2}=L_{\Phi 2} \cap L_{\Phi 1}$.

HistModif\&MorphProc CNNA2002 submitted to World Scientific : 4/26/2002 : 2:11 PM 


\section{Approximating the Histogram Modification PDEs}

By mapping the PDEs introduced in the previous section into nonlinear ODEs we consider two cases corresponding to (1), and (5) respectively. All these approximations could be implemented as a complex analogic algorithm based on an existing architecture [6].

Case 1: Contrast enhancement through histogram equalization

Based on (1) assuming $\alpha=0 ; H=N^{2} / M ; N^{2}=1 ; M=1$ :

$$
\frac{d \phi_{i j}(t)}{d t}=-\phi_{i j}(t)+\left(1-A_{i j}^{(1)}\right)
$$

Case 2: Contrast enhancement, denoising and embedded morphological processing

Based on (5) assuming $\alpha=1 ; \kappa=\operatorname{div}(\operatorname{grad}(\Phi)) ; H=N^{2} / M ; N^{2}=1 ; M=1$ :

$$
\frac{d \phi_{i j}(t)}{d t}=-\phi_{i j}(t)+\left(1-A_{i j}^{(2)}\right)+\frac{1}{4}\left(\phi_{i-1 j}(t)+\phi_{i+1 j}(t)+\phi_{i j-1}(t)+\phi_{i j+1}(t)\right)
$$

In all cases $A^{(1)}{ }_{i j}=A^{(2)}{ }_{i j}=$ const during the evolution [13], therefore should only be calculated once. Though $A_{i j}$ is the output of a global transformation it is possible to give an approximation based on purely local (analog and logic) operations. This "spatial decomposition" will be discussed in the sequel.

The following operational notations and definitions will be used throughout the algorithm descriptions (all templates referenced can be found in the CNN Software Library [16]):

Definition 1: Threshold - thresholds a gray-scale input image at a given gray-scale level. The output is a binary image defined as follows:

$$
\operatorname{Thr}\left(\Phi_{i j}, \lambda\right)= \begin{cases}1 & \text { if } \Phi_{i j}>\lambda \\ 0 & \text { otherwise }\end{cases}
$$

CNN implementation: by using the THRESH template.

Definition 2: Area - calculates the area measure corresponding to a given level-set of the input image. The output is a scalar defined as follows $\left(\Phi_{i j, B i n}\right.$ is the level-set calculated by the $T h r$ function):

$$
\operatorname{Area}\left(\Phi_{i j, B i n}\right)=\sum_{i=1}^{N} \sum_{j=1}^{N} \Phi_{i j, B i n}
$$

CNN implementation: by using the DIFFUS template the average value of an image can be calculated in the specified domain. If the initial state is a constant image (all pixels are set to +1 ) over the specified level-set then the diffusion output at steady-state gives a normalized area measure (a value in the range of $[0,1]$ ) of the level-set domain related to the entire image area.

Definition 3: Set level - sets the value of all pixels over a given mask to a specified gray-scale level. The output is a gray-scale image defined as follows:

$\operatorname{SetLev}\left(\Phi_{i j, \text { Bin }}, v\right)= \begin{cases}v & \text { if } \Phi_{i j, \text { Bin }}=1 \\ 0 & \text { otherwise }\end{cases}$

CNN implementation: by using the SETLEV template.

HistModif\&MorphProc_CNNA2002 submitted to World Scientific : 4/26/2002 : 2:11 
Definition 4: Erode - calculates erosion of a binary input image with a specified structuring element $B$. The set theoretical definition of the erosion based on Minkowski subtraction is as follows (- denotes translation):

$$
\operatorname{Erode}\left(\Phi_{i j, \text { Bin }}, B\right)=\Phi \otimes B=\cap\left\{\Phi_{i j, \text { Bin }}-b: b \in B\right\}
$$

CNN implementation: by using the EROSION template (single-step erosion) or PROPE (continuous erosion by a trigger-wave).

Definition 5: Dilate - calculates dilation of a binary input image with a specified structuring element $B$. The set theoretical definition of the erosion based on Minkowski addition is as follows (+ denotes translation):

$$
\text { Dilate }\left(\Phi_{i j, \text { Bin }}, B\right)=\Phi \oplus B=\cup\left\{\Phi_{i j, \text { Bin }}+b: b \in B\right\}
$$

CNN implementation: by using the DILATE template (single-step erosion) or PROPD (continuous dilation by a trigger-wave).

Definition 6: Norm - calculates a normalized version of a gray-scale input image. The formulation of the operation is as follows $\left(D_{\min }\right.$ and $D_{\max }$ stand for the minimum and maximum of the available dynamic range; $\Phi_{\min }$ and $\Phi_{\max }$ stand for the minimum and maximum of the input image, respectively):

$$
\operatorname{Norm}\left(\Phi_{i j}\right)=D_{\text {min }}+\frac{D_{\text {max }}-D_{\text {min }}}{\Phi_{\text {max }}-\Phi_{\text {min }}}\left(\Phi_{i j}-\Phi_{\text {min }}\right)=D_{\text {min }}+\frac{\Delta D}{\Delta \Phi}\left(\Phi_{i j}-\Phi_{\text {min }}\right)
$$

CNN implementation: first $\Phi_{\min }$ and $\Phi_{\max }$ is calculated by using the THRESH template and global logic (iterative). Since $\Delta D$ is known a priori, the implementation of (14) leads to an analogic algorithm based on template SCALE and simple arithmetics. Remark: the constant $b_{0}=\Delta D / \Delta \Phi$ that is the central element of the $B$ term in SCALE is image dependent, thus interaction with the digital environment is needed. It should be noted though that Norm is not an essential part of the histogram modification algorithm, it is included in the extended version (Algorithm 2, see later) in order to increase the robustness in a physical implementation with a limited analog precision (especially in case of very low input image dynamic range).

Definition 7: Diffuse - calculates a linear low-pass filtered version of a gray-scale input image. The formulation of the operation is as follows (* denotes convolution):

$$
\operatorname{Diff}\left(\Phi_{i j, 1}, \Phi_{i j, 2}\right)=\alpha \Phi_{i j, 1} * G_{1}\left(\sigma_{1}\right)+(1-\alpha) \Phi_{i j, 2} * G_{2}\left(\sigma_{2}\right)
$$

CNN implementation: equation (15) describes a homotopy in between two different linear convolutions by a Gaussian kernel. Under fairly mild conditions at some time $t$ this corresponds to the solution of a constrained diffusion type partial differential equation. After spatial discretization this can be mapped to a CNN structure ([8], [9]) programmed by template CDIFFUS. In this form the $A$ and $B$ term approximates $G_{1}$ and $G_{2}$, respectively. The filter characteristic is also dependent on the transient length $(t)$ of the CNN template.

The symbolic description of the histogram modification algorithm can be found in Fig. 1.

HistModif\&MorphProc_CNNA2002 submitted to World Scientific : 4/26/2002 : 2:11 


$$
\begin{aligned}
& \text { Algorithm } 1 \text { : } \\
& \Phi_{\lambda_{0}}=0, \quad A=N^{2} \\
& \text { for } i=1 \text { to } q \\
& \Phi_{\lambda_{i}}=\operatorname{Thr}\left(\Phi, \lambda_{i}\right) \\
& \bar{\Phi}_{\lambda_{i}}=\Phi_{\lambda_{i}} \text { xor } \quad \Phi_{\lambda_{i-1}} \\
& A_{i}=\operatorname{Area}\left(\Phi_{\lambda_{i}}\right) \\
& v=A_{i} / A \\
& \Phi_{\text {Map }}=\operatorname{SetLev}\left(\bar{\Phi}_{\lambda_{i}}, v\right) \\
& \Phi_{\lambda_{i-1}}=\Phi_{\lambda_{i}}
\end{aligned}
$$

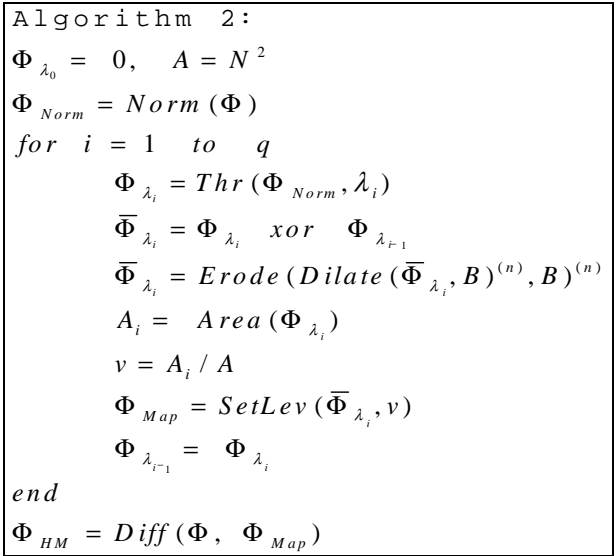

Fig. 1. Pseudo description of two versions of the histogram modification algorithm. Note, that in ALGORITHM 2 the order of erosion and dilation operations, defining a "multi-step closing", could also be inverted resulting in "multi-step opening".

AlgORITHM 1 and 2 show the implementation steps of (7) and (8) including all necessary calculations.

The CNN flow-chart of the generalized histogram modification analogic algorithm can be seen in Fig. 2.

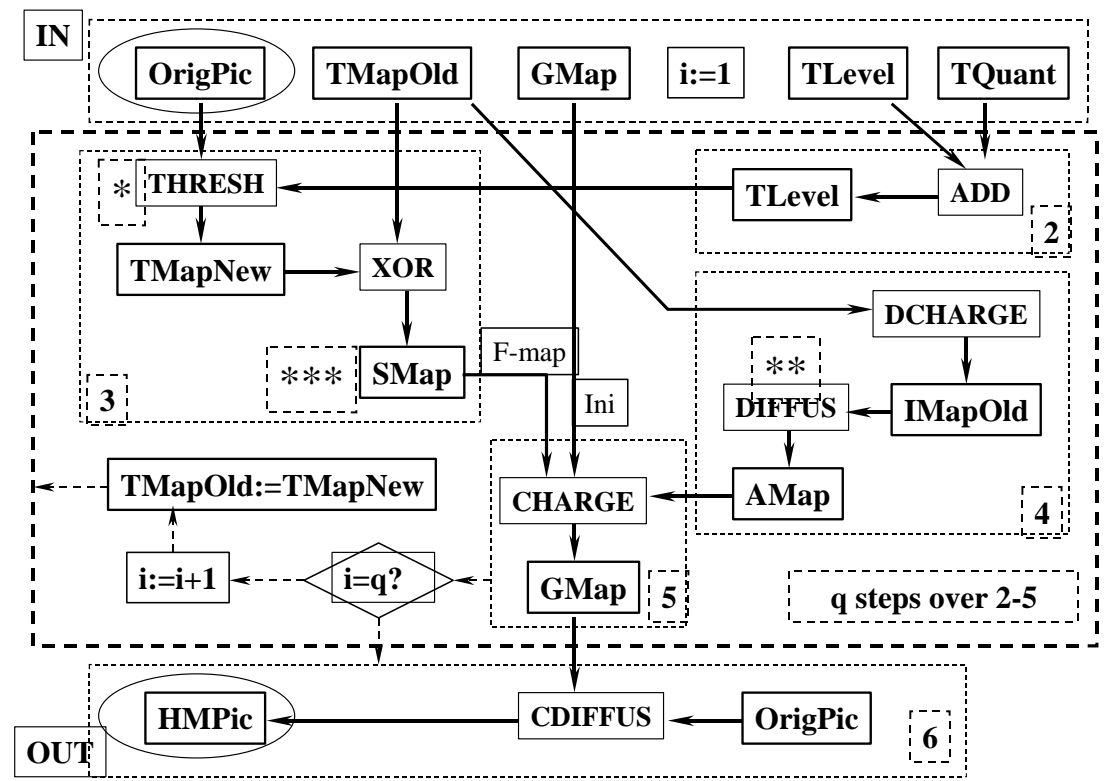

Fig. 2. The CNN analogic algorithm approximating the histogram modification PDEs discussed in Section 4. The processing stages are as follows: (1) Initialization, (2) Set threshold level, (3) Detect regions above threshold, (4) Calculate area measure, (5) Set Global Bias Map pixels, (6) Modify image histogram with noise suppression (regularization). Blocks 2-5 should be repeated q times (the number of gray-scale levels that specify the accuracy of the approximation). In the flowchart $* * *$, and $* * *$ mark the stages where the subroutines implementing further adaptive strategies can be embedded. Morphological operations are embedded at $* * *$ processing the level-set function SMap.

HistModif\&MorphProc_CNNA2002 submitted to World Scientific : 4/26/2002 : 2:11 $\mathrm{PM}$ 


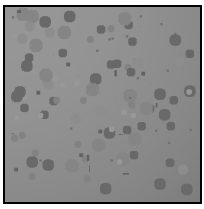

(a)

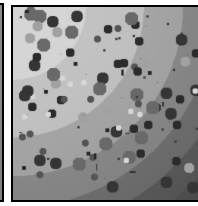

(b)

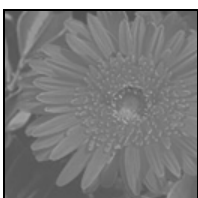

(c)

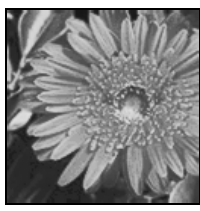

(d)

Fig. 3. Programmable global PDE on a cellular nonlinear architecture - simultaneous contrast enhancement with noise suppression: original, low contrast image $(a, c)$; enhanced - histogram equalized $(b, d)$.

$A^{(2)}{ }_{i j}$ in (8) differs from the first two versions, since it should include an embedded morphological processing [15]. Also, contrast stretching and further desirable smoothing and enhancement properties can be added to the algorithm, as will be discussed in the next section.

\section{Extended Algorithmic Schemes - Morphological Processing of the Level Sets}

An adaptive multi-thresholded output with shape enhancement can be obtained if a morphological processing is embedded at each gray-scale level considered. This could be implemented either through a multi-step erosion and dilation operations or using triggerwaves that approximate a continuous-scale binary morphology with flat structuring elements [7]. The description of the extended algorithm that contains the multi-step dilate-erode operations can be seen in Fig. 1 (Algorithm 2).

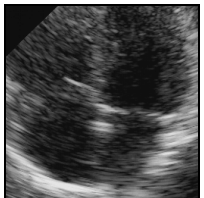

(a)

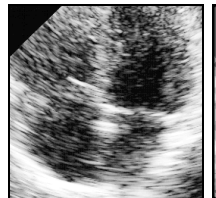

(b)

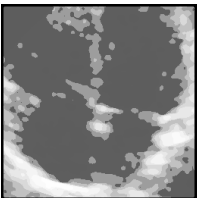

(c)

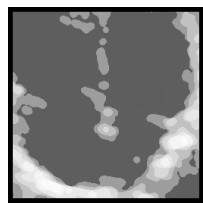

(d)

Fig. 4. Programmable global PDE on a cellular nonlinear architecture - simultaneous contrast enhancement and noise filtering with embedded morphological processing of the level-sets: (a) original, low contrast image, (b) histogram equalized image, (c)-(d) histogram modification with embedded differential morphological processing (implemented through expanding and shrinking trigger-wave fronts) at two different scales ( $3 \tau$ and $5 \tau)$ and 8 distinct gray-scale levels.

\section{Experimental results}

For comparative analysis we have implemented the above-discussed histogram modification method (Algorithm 2 without diffusion filtering, but including the morphological processing of the level-sets) in 6 different hardware-software configurations listed bellow (the $7^{\text {th }}$ complete CNN-UM chip implementation is still an on-going experiment).

Version 1: in MATCNN MATLAB Toolbox [17] simulating all analog CNN dynamics with an optimized C-code running on a CISC. $\mu$ P: Pentium 1 GHerz.

Version 2: as Version 1, except for simulating through the CNN fixed-points with an optimized C-code.

Version 3: as Version 2, except for the C-code contains the entire algorithm.

HistModif\&MorphProc_CNNA2002 submitted to World Scientific : 4/26/2002 : 2:11 PM 
Version 4: in Aladdin Professional with an optimized C-code that contains the entire algorithm running on a DSP. $\mu$ P: Texas TMS6x 250 MHerz.

Version 5: as Version 4, except for the morphology operation is optimized at the assembly level.

Version 6: as Version 4, except for the morphology operation is optimized for the CNN-UM chip. $\mu$ P: Alcatel ACE4k [6].

Version 7: in Aladdin Professional [18] running the entire algorithm on a CNN-UM chip (on-going). $\mu \mathrm{P}$ : Alcatel ACE4k [6].

Detailed measurement results can be seen in Fig. 5. Observe in Fig 5(b) that 7x2 binary morphology operations including logic are completed within $100 \mu$ s on the ACE4k CNN-UM chip.

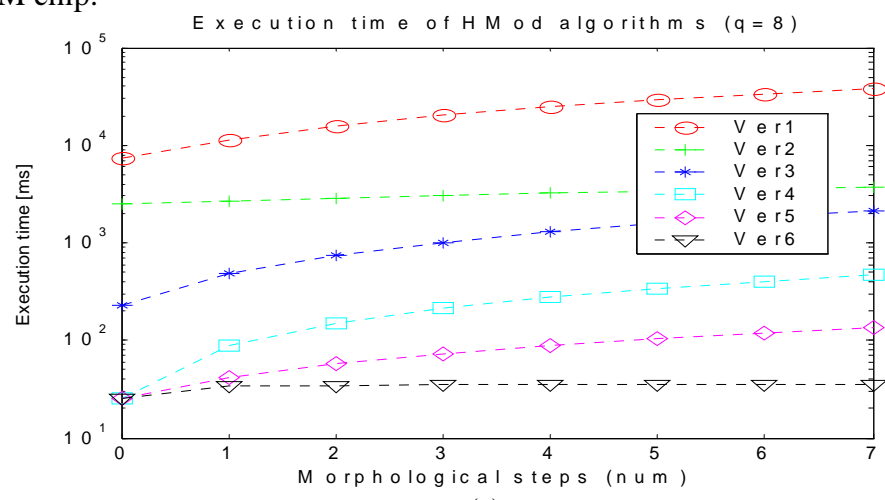

(a)

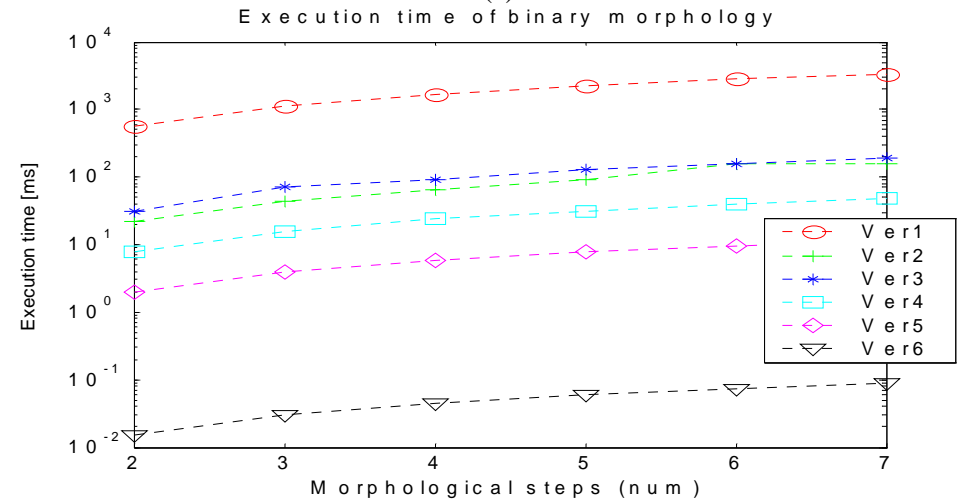

(b)

Fig. 5. Comparison of the implementations of the histogram modification algorithm (Ver1-Ver6) running on different hw-sw platforms.

\section{Conclusions}

In this paper we have shown how a PDE, designed for simultaneous contrast enhancement, noise suppression, and shape enhancement could be implemented as an analogic algorithm relying on purely local operations. We have also performed an exhaustive comparative experiment of different implementations on various hardware-

HistModif\&MorphProc_CNNA2002 submitted to World Scientific : 4/26/2002 : 2:11 PM 
software platforms with CISC, DSP and CNN-UM microprocessors. These measurements show how the efforts toward a fully parallel implementation (Version 7) of our algorithm could lead to an extremely efficient preprocessing scheme for various process real-time applications (possibly up to 1000 frames/sec with resolution of $64 x 64$, see the results for binary morphology in Fig 5(b)).

\section{References}

1. L. O. Chua and L. Yang, "Cellular Neural Networks: Theory and Applications", IEEE Trans. on Circ. \& Syst., Vol. 35, pp. 1257-1290, 1988.

2. L. O. Chua, and T. Roska, "The CNN Paradigm", IEEE Trans. on Circ. \& Syst., Vol. 40, pp.147-156, 1993.

3. T. Roska and L. O. Chua, "The CNN Universal Machine", IEEE Trans. on Circ. \& Syst., Vol. 40, pp. 163-173, 1993.

4. S. Espejo, R. Carmona, R. Domingúez-Castro, and A. Rodrigúez-Vázquez, "CNN Universal Chip in CMOS Technology", Int. J. of Circuit Theory \& Appl., Vol. 24, pp. 93-111, 1996.

5. Ari Paasio, Adam Dawidziuk, Kari Halonen and Veikko Porra, "Minimum Size 0.5 Micron CMOS Programmable 48 by 48 CNN Test Chip", in Proc. ECCTD '97, pp. 154-156, Budapest 1997.

6. S. Espejo, R. Domínguez-Castro, G. Liñán, Á. Rodríguez-Vázquez, “A 64×64 CNN Universal Chip with Analog and Digital I/O”, in Proc. ICECS'98, pp. 203-206, Lisbon 1998.

7. Cs. Rekeczky and L. O. Chua: "Computing with Front Propagation: Active Contour and Skeleton Models in Continuous-Time CNN", Journal of VLSI Signal Processing, Vol.23. No.2/3. pp.373-402, Kluwer, 1999.

8. Cs. Rekeczky, T. Roska, and A. Ushida, "CNN-based Difference-controlled Adaptive Nonlinear Image Filters", International Journal of Circuit Theory and Applications, Vol. 26, pp. 375-423, July-August 1998.

9. K. R. Crounse and L. O. Chua, "Methods for Image Processing in Cellular Neural Networks: A Tutorial", IEEE Trans. on Circ. and Sys., Vol. 42, pp. 583-601, 1995.

10. M. Csapody and T. Roska, "Adaptive Histogram Equalization with Cellular Neural Networks", in Proc. of CNNA 2002, pp. 81-86, Seville, 1996.

11. W. K. Pratt, Digital Image Processing, John Wiley \& Sons, pp. 275-284, 1991.

12. G. Sapiro and V. Caselles, "Histogram Modifications via Differential Equations", Journal of Differential Equations, Vol. 135, pp. 238-268, 1997.

13. G. Sapiro and V. Caselles, "Contrast Enhancement via Image Evolution Flows", Graphical Models Image Processing, Vol. 59, pp. 407-416, 1997.

14. V. Caselles, J-L. Lisani, and G. Sapiro, "Shape Preserving Local Histogram Modification”, IEEE Trans. on Image Processing, Vol. 8, pp. 220-230, 1999.

15. E. R. Daugherty, "An Introduction to Morphological Image Processing", SPIE Optical Engineering Press, 1992.

16. T. Roska, and L. Kék, ed. "Analogic CNN Program Library", Version 2.0, Technical Report, ANCL, Comp. and Aut. R. Inst of HAS, Budapest 2001.

17. Cs Rekeczky, "MATCNN - Analogic Simulation Toolbox for Matlab", Version 2.0, http://lab.analogic.sztaki.hu/MATCNN/ . Budapest 2002.

18. AnaLogic Computers Ltd: Aladdin Pro R2.1, http://www.analogic-computers.com/ Budapest 2002.

HistModif\&MorphProc_CNNA2002 submitted to World Scientific : 4/26/2002 : 2:11 